\title{
Shwachman-Diamond syndrome and solid tumors: 3 new patients from the French severe chronic neutropenia registry and literature review
}

Fares BOU MITRI ${ }^{1}$, Blandine BEAUPAIN ${ }^{1}$, Jean-Francois FLEJOU ${ }^{2}$, Matthieu PATIENT $^{3}$, Ilona OKHREMCHUCK ${ }^{3}$, Didier BLAISE ${ }^{4}$, Faézeh IZADIFAR-LEGRAND ${ }^{4}$, Jean-Alain MARTIGNOLES ${ }^{5}$, Francois DELHOMMEAU ${ }^{5}$, Christine Bellanné-Chantelot ${ }^{6}$, Jean-Francois EMILE ${ }^{7}$, and Jean Donadieu ${ }^{8}$

${ }^{1}$ Armand-Trousseau Childrens Hospital

${ }^{2}$ Hôpital Saint-Antoine

${ }^{3}$ HIA Sainte Anne

${ }^{4}$ Paoli-Calmettes Institute

${ }^{5}$ Hospital Saint-Antoine

${ }^{6}$ Hopital Universitaire Pitie Salpetriere

${ }^{7}$ Ambroise Pare Hospital Group

${ }^{8} \mathrm{CHU}$ Trousseau

October 15, 2020

\begin{abstract}
Shwachman-Diamond syndrome with Shwachman-Bodian-Diamond syndrome (SBDS) biallelic variants is a rare disorder that predisposes the carrier to malignant hemopathies but solid-cancer predisposition is poorly known. Among 155 cases entered in the French Severe Chronic Neutropenia Registry, three were identified original with malignant solid tumors (ovary, breast and esophagus). All cancers occurred in the fifth decade and, despite being at localized stages at diagnosis, they were rapidly fatal thereafter. No cancer was observed in the $14 \mathrm{HSCT}$ survivors post transplantation. Based on our experience and the literature, age $>40$ years was the only apparent risk factor.
\end{abstract}

\section{INTRODUCTION}

Shwachman-Diamond syndrome (SDS; OMIM 260400) is a rare autosomal-recessive multisystem disorder characterized by exocrine pancreatic dysfunction and neutropenia. ${ }^{1,2}$ It is mostly caused by biallelic germline variants in the Shwachman-Bodian-Diamond syndrome (SBDS ) gene. ${ }^{3}$ The disease's natural evolution is characterized by a long phase of mild neutropenia, which can evolve into aplastic anemia, myelodysplastic syndrome (MDS) and/or acute myeloid leukemia (AML) ( $35 \%$ by 30 years of age).$^{2,}{ }^{4}$ In addition, results of a recent study emphasized the relationship between tumor protein 53 (TP53) mutations and malignant hemopathies in SDS. ${ }^{5}$ Solid tumors have rarely been reported in SDS. Only four have been published so far: ovarian cancer, ${ }^{6}$ breast ductal adenocarcinoma, ${ }^{7}$ dermatofibrosarcoma ${ }^{8}$ and pancreatic adenocarcinoma ${ }^{9}$ (Table 1). Moreover, to date, no cohort study has estimated the solid-tumor rate or identified risk factors. That context incited us to report the French Severe Chronic Neutropenia Registry's experience.

\section{RESULTS and METHODS}

The Registry was initially created in 1993; since then, enrollment has been prospective. Full description 
of Registry methods is available elsewhere. ${ }^{2}$ The common definition of SDS was applied, ${ }^{10}$ and all patients reported carried a molecularly confirmed $S B D S$ mutation. The cutoff date was 31 May 2020. Cancers were classified according to the World Health Organization classification during a centralized review of the tumor samples (JFE) subjected to NGS analysis somatic study. Among the 155 patients included in this study [median follow-up: 16.4 years (2742 person-years)], three developed solid tumors during their monitoring. The cancer rate was $0 \%$ by the age of 40 years but increased dramatically during the $5^{\text {th }}$ decade (Figure 1). Their clinical histories are described. They were all followed according to published guidelines ${ }^{10}$ and none have presented, prior cancer diagnosis a myelodysplasia, and none have received GCSF prior cancer diagnosis.

\section{Patient \#5254}

A 3-month-old male was diagnosed with SDS. Because of recent dysphagia, at 48 years of age, he underwent esogastroduodenal endoscopy that revealed a moderately differentiated esophageal squamous cell carcinoma. The disease was localized without metastases. Treatment with a neoadjuvant radio- and chemotherapy (FOLFOX) preceded Mc Keown esophagogastrectomy. Histopathological studies showed a well-differentiated and mature squamous cell carcinoma limited to the muscle layer, without vascular, nerve or lymph-node involvement, and with healthy surgical margins (pT2N0M0). Four months later, during routine postoperative monitoring, he developed bilateral pleural effusions. Thoracentesis revealed a cancer-free chylous liquid. The thoracic duct was ligated laparoscopically due to unresponsiveness to medical treatment. One week later, the patient became febrile with acute respiratory distress. Pseudomonas stutzeri colonies were isolated from his left pleural effusion. Despite broad-spectrum antibiotics, G-CSF use, assisted ventilation and hemodynamic support, he died at the age of 49 due to multiorgan failure following a rapidly unfavorable course. NGS of cancer cells showed a pathogenic TP53 variant (c.638G >A; p.Arg213Gln), with an $18 \%$ variant-allele fraction (VAF), and a pathogenic HRAS mutation (c.175G>A; p.Ala59Thr), with a $5 \%$ VAF.

\section{Patient \#5726}

This woman has been followed for SDS since she was 10 years old. At 47, she was diagnosed with peritoneal carcinomatosis. Her initial signs were asthenia, diffuse abdominal pain, abdominal distension, anemia, and hypoalbuminemia with coagulation disorders (prolonged prothrombin time (PT) and partial thromboplastin time (PTT)). Histology of biopsies showed peritoneal adenocarcinoma in favor of a serous extension of an ovarian or primary peritoneal adenocarcinoma (BRCA1 and 2 negative). The extended work-up found multiple peritoneal nodules without hepatic, splenic, retroperitoneal, bone and cervical-thoracic localizations. Because of a potential paraneoplastic coagulopathy (PT $<40 \%$ and low coagulation factor concentrations, absence of circulating anti-VII autoantibodies), conventional chemotherapy (carboplatin-taxol) was prescribed, even though the tumor was resectable. She responded well to three chemotherapy cycles, followed by total hysterectomy with bilateral adnexectomy, omentectomy and peritonectomy, right colectomy, left colectomy and partial hepatectomy. Tumor histology revealed papillary and serous carcinoma of the peritoneum. The postoperative course was marked by severe infection. Ten days after surgery, she developed emphysematous cystitis with multifocal right pyelonephritis. No germs were identified. Despite respiratory assistance, empirical antibiotic therapy and G-CSF use, her situation deteriorated rapidly; she died 6 months after cancer diagnosis. NGS of cancer cells showed a TP53 pathogenic variant (c.329G >C, p.Arg110Pro) with $92 \% \mathrm{VAF}$, which suggests either variant homozygosity or deletion of the chromosome 17 which could not be studied in our sample.

\section{Patient \#5601}

Case 3 was a woman, diagnosed with unilateral breast cancer at the age of 43 . Her disease course was marked by a permanent neutropenia and thrombocytopenia. During a breast cancer screening, she was diagnosed with invasive ductal adenocarcinoma of the right breast: T1aN0, Her2-negative, estrogen- and progesterone-receptor-positive. She underwent partial mastectomy followed by radiation therapy with no significant complications. She died 11 months after her cancer diagnosis but no additional information are known about her death circumstances. 
The initial biopsy was not available for analysis NGS analysis.

\section{DISCUSSION}

We described three SDS patients who developed solid tumors. Table 1 summarizes their cases and four others previously published. ${ }^{6-9}$ All the cancers in our series occurred during the fifth decade (median: 47 years), whereas the published cases of cancers in SDS patients appeared slightly earlier (median: 34.5 years; range: $17-43$ years). Five (71.4\%) of the seven patients were women, two with breast adenocarcinoma and two with ovarian tumors. All reported cases had localized or locally advanced disease at diagnosis.

The overall cancer rate for SDS patients in our Registry was 1.9\% (3/155); cancer development was clearly age-related, because all occurred between 40 and 60 years of age. That rate is not as high as that for malignant hemopathies, ${ }^{2}$ but the SDS-cancer relationship remains a worrying observation. Age was the sole risk factor identified. None of these cancers was preceded by exposure to chemotherapy, radiotherapy, immunosuppressive agents or G-CSF therapy. Notably, among the 21 SDS HSCT patients in our 155-patient cohort, 14 survived $>5$ years without any diagnosed solid tumor. That observation contrasts with another model of inherited bone-marrow failure like Fanconi anemia. The latter is characterized by the occurrence of solid ENT cancers (head \& neck squamous cell carcinoma) after chemotherapy, in particular post-HSCT. ${ }^{11}$

The care of the cancer is another concern in SDS patients as their overall survival lasted $<1$ year, and death was attributed to cancer progression or therapeutic complications. Patient \#5726 and patient \#5254 received lower first-line treatment doses because of preexisting neutropenia or thrombocytopenia. These observations emphasize the difficulty to manage solid cancers in patients with SDS despite the adaptation of cancer therapeutic guidelines.

Lastly, tissue NGS study in the 2 patients which can be studied demonstrated the presence of a TP53 pathogenic variant. Pertinently, the SBDS mutation contains an escape mechanism consisting of TP53 activation that maintains protein synthesis. Those gain-of-function variants may generate malignant clones emerging leading to malignant hemopathies. ${ }^{12}$ The pathogenic TP53 variants found in \#5254 and \#5726 raise the possibility of a hypothetical common mechanism both in SDS hematological clonal evolution as well as in tissue malignancies ${ }^{13,14}$. Further molecular and cellular studies are needed to understand this complication in the natural history of SDS in order to treat it ${ }^{15}$.

Conflict of Interest: none

Acknowledgements: The French registry is supported by grants from Inserm and by a grant from the 111 les arts. This project is supported by constant and unlimited support of the Association Sportive de Saint Quentin Fallavier since 2004 with the unlimited commitment of Mr Gonnot. The authors thank the association IRIS for her support.

\section{REFERENCES}

1. Cesaro S, Pegoraro A, Sainati L, et al. A Prospective Study of Hematologic Complications and Long-Term Survival of Italian Patients Affected by Shwachman-Diamond Syndrome. J Pediatr 2020:219:196-201.

2. Donadieu J, Fenneteau O, Beaupain B, et al. Classification of and risk factors for hematologic complications in a French national cohort of 102 patients with Shwachman-Diamond syndrome. Haematologica 2012:97:1312-1319.

3. Warren AJ. Molecular basis of the human ribosomopathy Shwachman-Diamond syndrome. Adv Biol Regul 2017.

4. Myers KC, Furutani E, Weller E, et al. Clinical features and outcomes of patients with ShwachmanDiamond syndrome and myelodysplastic syndrome or acute myeloid leukaemia: a multicentre, retrospective, cohort study. Lancet Haematol 2020:7:e238-e246.

5. Xia J, Miller CA, Baty J, et al. Somatic mutations and clonal hematopoiesis in congenital neutropenia. Blood 2018:131:408-416. 
6. Alter BP, Giri N, Savage SA, Rosenberg PS. Cancer in the National Cancer Institute inherited bone marrow failure syndrome cohort after fifteen years of follow-up. Haematologica 2018:103:30-39.

7. Singh SA, Vlachos A, Morgenstern NJ, et al. Breast cancer in a case of Shwachman Diamond syndrome. Pediatr Blood Cancer 2012:59:945-946.

8. Sack JE, Kuchnir L, Demierre MF. Dermatofibrosarcoma protuberans arising in the context of Shwachman-Diamond syndrome. Pediatr Dermatol 2011:28:568-569.

9. Dhanraj S, Manji A, Pinto D, et al. Molecular characteristics of a pancreatic adenocarcinoma associated with Shwachman-Diamond syndrome. Pediatr Blood Cancer 2013:60:754-760.

10. Dror Y, Donadieu J, Koglmeier J, et al. Draft consensus guidelines for diagnosis and treatment of Shwachman-Diamond syndrome. Ann N Y Acad Sci 2011:1242:40-55.

11. Masserot C, Peffault de LR, Rocha V, et al. Head and neck squamous cell carcinoma in 13 patients with Fanconi anemia after hematopoietic stem cell transplantation. Cancer 2008:113:3315-3322.

12. Donadieu J, Delhommeau F. TP53 mutations: the dawn of Shwachman clones. Blood 2018:131:376-377.

13. Goh AM, Coffill CR, Lane DP. The role of mutant p53 in human cancer. J Pathol 2011:223:116-126.

14. Golomb L, Volarevic S, Oren M. p53 and ribosome biogenesis stress: the essentials. FEBS Lett 2014:588:2571-2579.

15. Green JA, Von EM, Abrahmsen LB. Restoration of conformation of mutant p53. Ann Oncol 2018:29:1325-1328.

Figure Legend

FIGURE 1 Kaplan-Meier estimation of the risk of solid tumor with 95\% confidence interval among 155 patients with SBDS Shwachman syndrome.

\section{Hosted file}

Solid tumors in SDS_Table.pdf available at https://authorea.com/users/367454/articles/486875shwachman-diamond-syndrome-and-solid-tumors-3-new-patients-from-the-french-severe-

chronic-neutropenia-registry-and-literature-review 


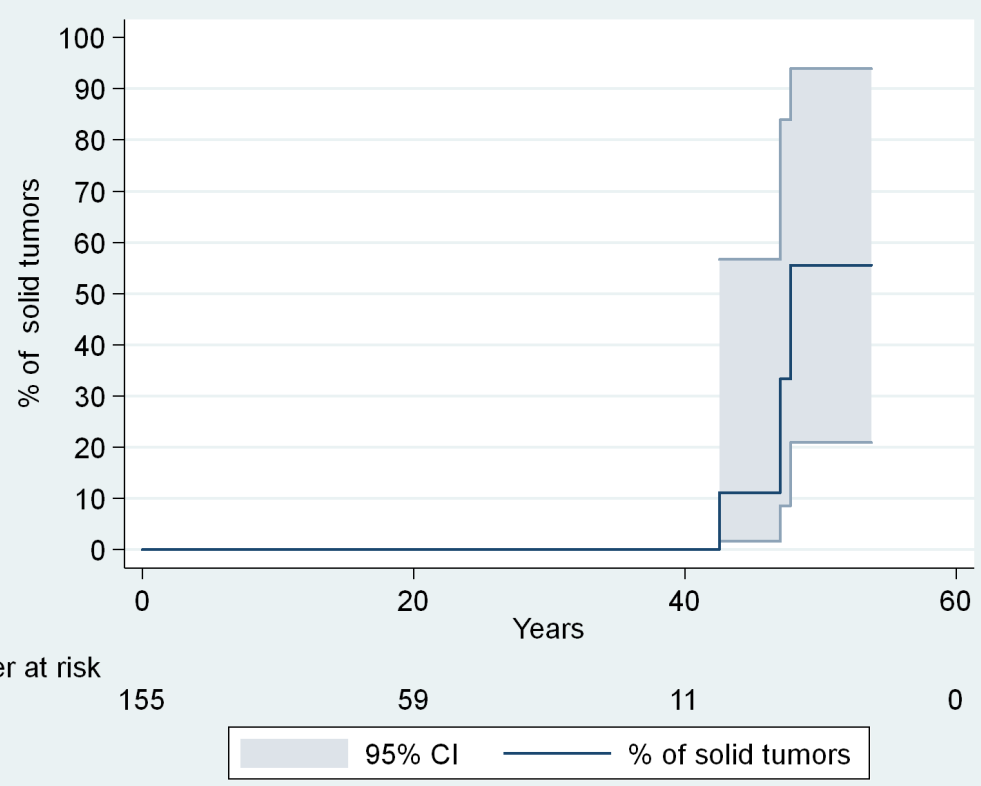

